psychopraxis.neuropraxis 2014 - 17:1-2

DOI 10.1007/s00739-014-0199-9

Online publiziert: 23. Oktober 2014

๑) Springer-Verlag Wien 2014

\section{Sehr geehrte Leserinnen! Sehr geehrte Leser!}

Die Zeitschrift psychopraxis.neuropra$x i s$ hat es sich zur Aufgabe gemacht, Falldarstellungen und Beiträge zu publizieren, die für die Praxis Relevanz haben. Falldarstellungen von komplexen Krankheitsbildern oder längerfristigen Behandlungsverläufen haben eine wichtige Funktion in der Ergänzung zu groß angelegten Studien oder Metaanalysen. Gelten Metaanalysen und kontrollierte randomisierte Studien als die höchstmögliche Evidenzstufe, so muss klar sein, dass dies nur für eng umschriebene Bereiche gilt. Ein- und Ausschlussregelungen in einzelnen Studien oder im Falle von Metaanalysen für Studien lassen zwar Aussagen mit geringerem Bias zu, die Generalisierbarkeit dieser Aussagen leidet aber darunter. Um diese Aussagen mit hoher Evidenz aber geringer Generalisierbarkeit auch auf komplexere klinische Situationen extrapolieren zu können, sind klinische Erfahrungen, also Falldarstellungen besonders hilfreich. In diesem Sinne stellen auch Fallberichte eine wichtige Grundlage für unser klinisches Handeln dar.

Eine wichtige Aufgabe der Psychiatrie ist es, psychische Phänomene zu benennen, bei Dysfunktionalität in Syndrome einzuordnen und schließlich im Idealfall mit einer Diagnose einen geeigneten Behandlungspfad einzuschlagen.

Viele dieser "Wortschöpfungen“" aus dem Bereich der Psychiatrie (Hypochondrie, Idiot etc.) werden zu Schimpfworten. Umgekehrt können Spezialbegriffe von psychischen Beschwerden aber auch eine enorme Popularität erlangen. Mit der regelmäßigen Überarbeitung der Diagnosekriterien und der Einarbeitung aktueller wissenschaftlicher Erkenntnisse erscheint in regelmäßigen Abständen eine neue Version des DSM (Diagnostisches Statistisches Manual der Amerikanischen Psychiatrischen Association), zuletzt im Mai 2013 die 5. Version, (die deutsche

\title{
Brief des Herausgebers
}

\section{Grundlage für klinisches Handeln}

Version wird für Herbst 2014 erwartet), und des Kapitels F der ICD der WHO (Kapitel für psychische Störungen der International Classification of Diseases).

So wurde zum Beispiel im DSM-5 der Begriff Hypochondrie aufgrund seiner stigmatisierenden Konnotation weggelassen und durch den Begriff „Illness Anxiety Disorder“ ersetzt. Geradezu zum Modebegriff geworden ist das „Burn-out", das mit dem arbeitspsychologischen Burnout-Syndrom oft nur mehr wenig zu tun hat. Für die Praxis könnte „Burn-out“, wie es in der Bevölkerung verwendet wird, so übersetzt werden: „Mir geht es psychisch nicht gut. Ich bin ein fleißiger Mensch und möchte nicht stigmatisiert werden." Welches Syndrom oder welche psychiatrische Diagnose dahinter steht, muss in den meisten Fällen noch eruiert werden. Ist es nur eine Erschöpfung in Zusammenhang mit Überarbeitung, dann kann vom Burn-out-Syndrom gesprochen werden. Kommen jedoch noch weitere Symptome dazu, wie zum Beispiel depressive Stimmung, Freud- und Interesselosigkeit, dann könnte schon eine Depression dahinter stecken. Im klinischen Alltag findet sich eine breite Palette an psychischen Störungen, wenn die Patient(inn)en von „Burn-out“ sprechen. Wichtig ist, mit der richtigen psychiatrischen Diagnose auch die optimalen therapeutischen Schritte einzuleiten.

\section{》) Wichtig ist, mit der richtigen psychiatrischen Diagnose auch die optimalen therapeutischen Schritte einzuleiten}

Obwohl die Diagnosemanuale immer umfangreicher werden, gibt es noch immer einen Mangel an Begriffen für psychische Phänomene. Bei dissoziativen Phänomenen hat die Bevölkerung häufig gar keine geeigneten Ausdrücke zur Verfügung, um diese Zustände zu beschreiben. Bei Alltagsdissoziationen steht oft nicht einmal ein entsprechendes Substantiv zur Verfügung, es muss auf eine Beschreibung des „Tuns“ zurückgegriffen werden, z. B. „in’s Narrnkastl schaun“ (quasi eine allgemeine pensée opératoire).

$\mathrm{Da}$ die veränderten Bewusstseinszustände mangels geeigneter Worte schwer zu beschreiben sind, gehen sie in einer Exploration meist unter bzw. können auch fehlinterpretiert werden. Hier ist es Aufgabe der Psychiatrie, alltagstaugliche Begriffe zur Verfügung zu stellen, um besser darüber sprechen zu können. Nicht nur für den Bewusstseinszustand „Schlaf“ bedarf es eines Substantivs, sondern auch für die vielen anderen Bewusstseinszustände, die wir kennen. Ansätze dafür gibt es bereits, wenn „a-Zustand“ oder „FloatingZustand“ für bestimmte Entspannungszustände in Wellnesskatalogen auftauchen und von der Allgemeinbevölkerung offensichtlich auch angenommen werden.

Der Begriff „a-Zustand“ wurde mithilfe des EEGs entwickelt. Entsprechend der Grundfrequenz, die das Gehirn erzeugt und die mit dem Elektroenzephalogramm aufgezeichnet wird, lassen sich Bewusstseinszustände abgrenzen. So ist der „a-Zustand“ ein leichter Entspannungszustand $(8-12 \mathrm{~Hz})$ und das Alltagsbewusstsein ein , $\beta$-Zustand“ (13-21 Hz). Um dissoziative Bewusstseinszustände besser explorieren zu können, braucht es eine Verbreitung einschlägiger Termini und eine klare Vorstellungen über dissoziativen Bewusstseinsveränderungen in der Bevölkerung.

Die Psychiatrie steht somit in einem Spannungsfeld von Krankheitsdefinition und der Definition von Verhaltensmustern ohne Krankheitswert. Da Krankheit in der Gesellschaft auch mit „Gratifikationen“ einhergeht, ist die Stigmatisierung ein Begleiter von unscharf definierten oder schwer verständlichen Krankheitsbildern. Um psychische Störungen aus dem Stigmabereich herauszubringen, braucht es valide Krankheitsdefinitionen und ein Verständnis dieser Störungsbilder in der Bevölkerung. Von einem op- 


\section{Editorial}

timalen Zustand sind wir hier noch weit entfernt.

Krankheiten können von der Medizin nur dann erfolgreich behandelt werden, wenn es auch in der Allgemeinbevölkerung ein gutes Verständnis darüber gibt. So haben wir ein Zeitalter der Infektionskrankheiten mit Seuchen in Europa gehabt. Mit der Entwicklung des Verständnisses des Prinzips „Infektion“ konnten viele Maßnahmen getroffen werden, um Infektionskrankheiten in den Griff zu bekommen. Ähnlich könnte heute von einem Zeitalter der neuropsychischen oder biopsychosozialen Störungen gesprochen werden. Das Verständnis des Prinzips „Selbstorganisation“ könnte möglicherweise in ähnlicher Weise dazu füh- ren, dass viele dieser Störungsbilder besser behandelt werden können.

Mit der Entwicklung von neuen Diagnosesystemen wird versucht, dieser Aufgabe Rechnung zu tragen. Dennoch genügt eine reine deskriptive Diagnostik, wie sie im ICD-10 oder im DSM-5 vorliegt, nicht. Es braucht auch ein Verständnis für die dahinter liegenden Prozesse. Ansätze dafür bietet ein relativ neues Klassifikationssystem: RDoC (Research Domain Criteria).

Fallberichte haben hier die Funktion, komplexe psychische Zustandsbilder auf die aktuellen Diagnosesysteme zu beziehen, eventuell auch Prozesse aufzuzeigen, die hinter diesen Diagnosen liegen und erfolgreiche längerfristige Behandlungs- pfade aufzuzeigen, die nicht Gegenstände von Studien sein können.

In diesem Sinne wünsche ich Ihnen eine gewinnbringende Lektüre der $p s y c h o p$ raxis.neuropraxis für Ihre klinische Praxis.

Martin Aigner

\section{Korrespondenzadresse}

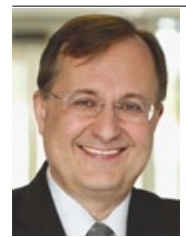

Prim. Privatdozent Dr. M. Aigner

Abteilung für Erwachsenenpsychiatrie Universitätsklinikum Tulln Alter Ziegelweg 10, 3430 Tulln martin.aigner@tulln.lknoe.at

\section{Hier steht eine Anzeige.}

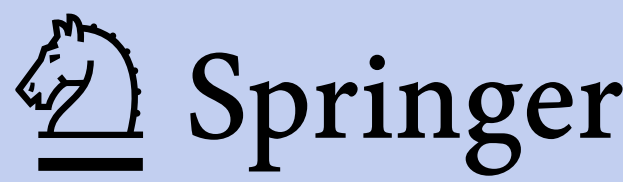

